\title{
A TIME-FREQUENCY CHARACTERIZATION FRAMEWORK FOR SIGNALS ISSUED FROM UNDERWATER DISPERSIVE ENVIRONMENTS
}

\author{
A. Jarrot ${ }^{\dagger}$, C. Ioana ${ }^{\ddagger}$, C. Gervaise ${ }^{\dagger}$, A. Quinquis ${ }^{\dagger}$ \\ ${ }^{\dagger} \mathrm{E}^{3} \mathrm{I}^{2}$ Laboratory (EA 3876) - ENSIETA, \\ 2 Rue François Verny, 29806, Brest, FRANCE \\ phone: +33(0) 298348720 - fax: +33(0) 298348750 \\ emails: [jarrotar, gervaice, quinquis]@ensieta.fr \\ ‡ LIS Laboratory \\ BP 46, 961 rue de la Houille Blanche \\ 38402 Saint-Martin d'Hères cedex, FRANCE \\ phone: +31(0) 476826422 -fax: +33(0) 476826384 \\ email: cornel.ioana@lis.inpg.fr
}

\begin{abstract}
Time-frequency representations constitute the main tool for analysis of non-stationary signals arising from environmental systems. Recently, the interest for underwater dispersive channels appears since dispersivity phenomena act at very low frequencies which are well suited for long range underwater communication. In such a case, a main interest is to perform estimation of the impulse response of such channel for processing purposes. In this paper we introduce a time-frequency analysis tool that aims to extract the time-frequency components of the channel impulse response. This technique is based on the adaptive time-frequency filtering whose parameters are defined by a local chirp matching procedure. Tests provided for realistic scenarios illustrate the potential and the benefits of the proposed approach.
\end{abstract}

Index Terms - Time-frequency analysis, System identification, Dispersive channels.

\section{INTRODUCTION}

Considering the general non-stationary behavior of the observations encountered in real applications, analysis in the field of time-frequency domain constitutes the best suited technique to identify the relevant structures for information processing [1]. In the context of underwater acoustic, considering the dispersive behavior of channels has fundamental interest, especially in the case of systems operating at low frequencies. In [2] a theoretical description of signal issued from underwater dispersive channels is described. It shows that dispersivity phenomena introduce non-linear time-frequency deformations of emitted signals. In addition, such deformations depend on the propagation path, and leads to a multi-component heterogeneous non-linear time-frequency behavior of the received signal. Moreover, coefficients loss and time-frequency proximity of paths bring the signal model very complex. As shown in [1], [2], analysis of dispersive underwater signals by typical time-frequency methods is a challenging problem.

In this paper, a characterization framework that aims to deal with signals issued from underwater dispersive environment is proposed. The high-resolution requirement and the non-stationarity of all signals imply the construction of a new time-frequency analysis strategy. A two steps solution is proposed. First step consists in roughly modeling the instantaneous frequency of each component as a set of local chirps. This model is established by finding the best matched local chirp to the component. Unlike conventional approaches, the first step exploits the initial phase of the local chirps, demonstrating the importance of this parameter. Second step exploits this estima-

tion to design the time-frequency filter for the extraction of the corresponding part of the signal. Finally, this procedure is performed again for remaining components. The paper is structured as follows. In section 2, the method is described in details. In section 3, after a short presentation of the underwater dispersive channels, the potential of the proposed approach for some realistic data is proved. We conclude in section 4 .

\section{METHOD}

In a large number of applications (radar, sonar, underwater acoustic), signals modeling is classically done by means of a multi-component coherent time-frequency structures model. Such a model can be expressed by :

$$
x(t)=\sum_{m=1}^{M} x_{m}(t)+n(t),
$$

where $n(t)$ is the noise and where each component is expressed as $x_{m}(t)=A_{m}(t) \cos \left(\phi_{m}(t)\right)$. With this notations, $A_{m}(t)$ and $\phi_{m}(t)$ are respectively the instantaneous amplitude and the instantaneous phase of the $m^{\text {th }}$ component. In what follows, we assume that each $A_{m}(t)$ is slowly varying compared to $\phi_{m}(t)$. In addition, we assume that the derivative of $\phi_{m}(t)$ is a continuous function.

Let the signal $x(t)$ be the received signal that has to be characterized. In this context, the problem is to find the set $\left\{A_{m}(t), \phi_{m}(t)\right\}$ such that

$$
\hat{x}(t)=\underset{\left\{A_{m}(t), \phi_{m}(t)\right\}}{\operatorname{argmin}} d(x(t), \hat{x}(t)),
$$

where $d(x(t), \hat{x}(t))$ is a measure between the received signal and the model.

In the case of unknown model, the shapes $A_{m}(t)$ and $\phi_{m}(t)$ are unknown. Thus, due to the extreme variability of these parameters, minimization of (2) is untractable, except for very simples cases. To solve this problem we propose a general framework that aim to estimate the set $\left\{\hat{A}_{m}(t), \hat{\phi}_{m}(t)\right\}$ without prior knowledge on the model.

\subsection{General methodology}

A broad class of characterization methods has been proposed for non-stationary mono-component signals (e.g. polynomial modelization of phase [3], chirplets [4],...). However, it is well-known that such approaches lead to very high difficulties in the case of a heterogenous mixture of close time-frequency components.

To overcome this difficulties we consider, in this paper, the general framework illustrated in Fig. 1. It is a recursive structure based 


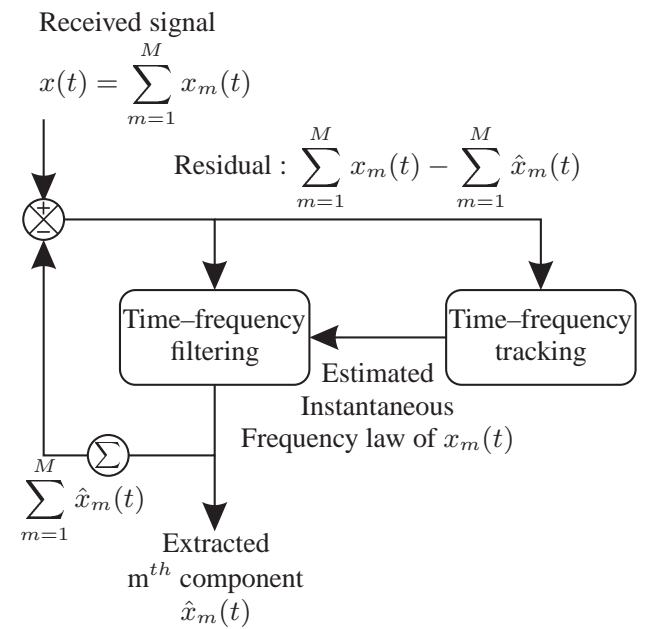

Fig. 1. General principle of the proposed characterization framework.

on two main steps: the time-frequency tracking step, and the timefrequency filtering step which are described next.

\subsection{Time-frequency tracking}

The goal of this step is to estimate, from the mixture $x(t)$, the instantaneous frequency law of the $m^{t h}$ component $x_{m}(t)$. The overall tracking strategy is illustrated illustrated in Fig. 2.

\subsection{1. mono-component case}

Let us first consider the case when the received signal is a monocomponent signal such that $x(t)=x_{m}(t)$. We assume that $x_{m}(t)$ can be approximated on each time segment $\Delta t$ by a linear chirp $\bar{x}_{m}(t)$. Based on this assumption, $\bar{x}_{m}(t)$ can be expressed on each time segment $t \in\left[j \Delta t,(j+1) \Delta t\left[\right.\right.$ by $\bar{x}_{m, j}(t)=\cos \left(\bar{\phi}_{m, j}(t)\right)$ where

$$
\bar{\phi}_{m, j}(t)=a_{j}+b_{j} t+c_{j} t^{2}
$$

This local chirp model has been extensively considered in addition with basis pursuit technics [4] to provide sparse representations of signals. However, basis pursuit technics generally consider independently each chirp which is not well-suited in our context : since the instantaneous phase of $x_{m}(t)$ is a continuous function, one can expect that the chirp $\bar{x}_{m, j}(t)$ is "connected" with the chirp $\bar{x}_{m, j+1}(t)$.

While the match filtering using reference signals (e.g. chirps) is a traditional technique in radar or sonar [1], its interest in timefrequency analysis has been materialized by a local match filtering procedure [2]. In the remainder of this paper, we suggest to improve the classical matched filtering approach by requiring continuity of the initial phase between chirps $\bar{x}_{m, j}(t)$ and $\bar{x}_{m, j+1}(t)$.

This can be interpreted as requiring following continuity constraints

1 Chirp sequence has continuous instantaneous frequency

$$
b_{j+1}=b_{j}+2(j \Delta t)\left(c_{j}-c_{j+1}\right) .
$$

2 Chirp sequence has continuous instantaneous phase

$$
a_{j+1}=a_{j}+b_{j}(j \Delta t)+c_{j}(i \Delta t)^{2} .
$$

Let $\bar{x}_{m, 0}(t)$ be the initial chirp that best approximate the component $x_{m}(t)$ over the time interval $\left[0, \Delta t\left[\right.\right.$. The next chirp $\bar{x}_{m, 1}(t)$ on

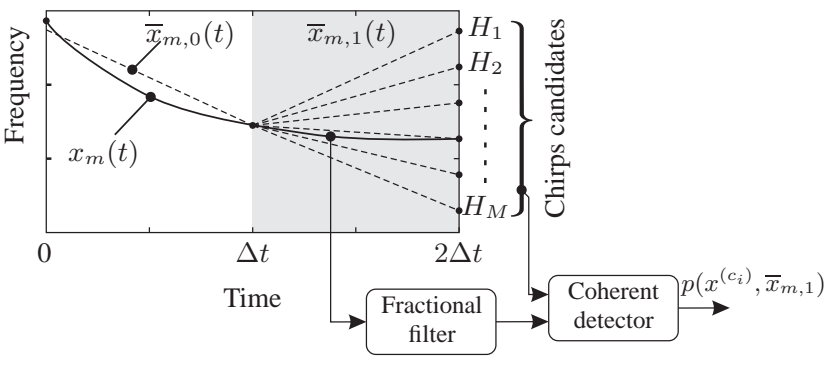

Fig. 2. Illustration of the time-frequency grouping strategy. The search of the next segment can be reformulated as a multi-hypothesis coherent detection problem.

the time-interval $[\Delta t, 2 \Delta t[$ is defined to be the one that best match $x_{m}(t)$ with regards to the phase constraints (4) and (5).

We formulate this problem as a multi-hypothesis detection problem where $H_{1}, \ldots, H_{M}$ are the chirp candidates. The problem of finding the best candidate can be handled by testing all hypotheses $H_{M}$ and choosing the one for which the match between the candidate and the true signal maximizes some criteria.

The problem of finding the best candidate when the match depends on amplitude and phase of signals is generally refereed as a coherent detection problem [6] and can be solved by a large variety of techniques. In [7], authors suggest to use the so-called quadrature matched filtering. This approach consists in comparing the signal $x_{m}(t)$ with in phase and in quadrature waveforms such that

$$
\begin{aligned}
& x_{c}=\int_{j \Delta_{i}}^{(j+1) \Delta_{i}} x_{m}(t) \cos \bar{\phi}_{m, j}(t) d t \\
& x_{s}=\int_{j \Delta_{i}}^{(j+1) \Delta_{i}} x_{m}(t) \sin \bar{\phi}_{m, j}(t) d t
\end{aligned}
$$

and to calculate the penalty criteria $p\left(x_{m}(t), \bar{x}_{m, j}\right)$

$$
p\left(x_{m}, \bar{x}_{m, i}\right)=\frac{n_{s} x_{c}^{2}-2 n_{c s} x_{c} x_{s}+n_{c} x_{s}^{2}}{2 n_{c} n_{s}-2 n_{c s}^{2}},
$$

with $n_{c}=\int_{j \Delta_{i}}^{(j+1) \Delta_{i}} \cos ^{2} \bar{\phi}_{m, j}(t) d t, n_{s}=\int_{j \Delta_{i}}^{(j+1) \Delta_{i}} \sin ^{2} \bar{\phi}_{m, j}(t) d t$ and $n_{c s}=\int_{j \Delta_{i}}^{(j+1) \Delta_{i}} \cos \bar{\phi}_{m, j}(t) \sin \bar{\phi}_{m, j}(t) d t$. Thus, the penalty criterion $p\left(x_{m}(t), \bar{x}_{m, j}\right)$ is maximized for $x_{m}(t)=\bar{x}_{m, j}$ gives optimal estimation if $n(t)$ is a white Gaussian noise.

\subsection{2. multi-component case}

In practice, the received signal $x(t)$ is a multi-component signal. In this case, the white Gaussian noise is clearly not satisfied because of the multi-component nature of $x(t)$.

For this reason, we suggest to introduce a preprocessing step in the quadrature matched filtering to isolate the specific time-frequency band on which the chirp candidate is defined. Since the chirp candidate is a linear modulation of frequency, we suggest to use the class of linear filters based on the Fractional Fourier transform described in [8] to isolate the specific time-frequency band around the instantaneous frequency of the chirp candidate. We define as $x^{\left(c_{i}\right)}(t)$ the signal that is obtained by filtering $x(t)$ around the instantaneous frequency of $\bar{x}_{m, j}$. This signal $x^{\left(c_{i}\right)}(t)$ is given by :

$$
x^{\left(c_{i}\right)}(t)=\mathcal{F}^{\tan ^{-1}\left(c_{i}\right)}\left(\left(\mathcal{F}^{-\tan ^{-1}\left(c_{i}\right)} x\right)(f) \cdot H(f)\right)(t),
$$


where $\mathcal{F}^{\alpha}$ is the fractional Fourier transform of angle $\alpha$, and $H(f)$ is the frequency response of a pass-band filter.

From the estimation $\bar{x}_{m, 1}$, we repeat this procedure for all time segments $j \Delta t$ to obtain the sequence $\left\{\bar{x}_{m, 0}(t), \ldots, \bar{x}_{m, J}(t)\right\}$.

\subsection{Time-frequency filtering}

The goal of the time-frequency filtering step, is to extract from the received signal $x(t)$ the component $x_{m}(t)$ that has be tracked in the previous tracking step.

Let $w(t)$ be a continuous strictly increasing function. We define the local harmonic convolution operator [5] between the signal $x(t)$ and the filter function $h(t)$ by :

$$
x(t) \stackrel{w(t)}{*} h(t)=\int_{\mathbb{R}} \frac{d w(\tau)}{d \tau} x(\tau) h\left(w^{-1}(t)-w^{-1}(\tau)\right) d \tau .
$$

It can be noticed that in the special case where $w(t)=t$, the classical convolution operator is recovered. Based on this operator, the linear time-invariant filtering theory is extended to a more general class of linear filters that is valid for non-stationary signals.

The general principle of this class of filters is depicted in Fig. 3. Its aim is to decompose the received signal $x(t)$ into two signals $x_{L}(t), x_{H}(t)$ such that each signal is contained in a specific timefrequency region delimited by a time-varying cutoff frequency $f=$ $e(t)$.

It can be shown that such a decomposition can be obtained by processing the signal by means of (10) if the following conditions are fulfilled [5]

1. $w(t)$ function matches the time-varying cutoff frequency such that

$$
w(t)=f_{0}\left[\int_{-\infty}^{t} e(u) d u\right]^{-1}, \quad f_{0} \in \mathbb{R}^{+}
$$

2. filter functions $h_{L}(t)$ and $h_{H}(t)$ are respectively lowpass and highpass filters, with zero-phase and with a cutoff frequency equals to $f_{0}$.

Thus, from the chirp sequence $\left\{\bar{x}_{m, 0}(t), \ldots, \bar{x}_{m, J}(t)\right\}$ we generate the estimated instantaneous frequency $I F(t)$ of the component $x_{m}(t)$ by B-spline interpolation. Then, the the extraction of the $k^{t h}$ component is performed with two filters that have time-varying cutoff frequency $e(t)=I F(t) \pm \Delta f$ where $2 \Delta f$ is the band of the time-varying passband filter.

\section{NUMERICAL EXAMPLES}

In this section, performances of the proposed characterization framework is illustrated in the context of underwater dispersive channel. For this purpose, we first introduce the considered signal model and then illustrate performances of the method on a realistic simulation.

\subsection{Underwater dispersive channels}

From a signal processing point of view, the underwater channel is characterized by a non-linear time-frequency distortion of the emitted signal which depends on two effects [9]:

- Attenuation due to the reflection to the bottom and see surface;

- Non-linear group delay, having distinct characteristics for each path, which produces a different delay for each spectral component.

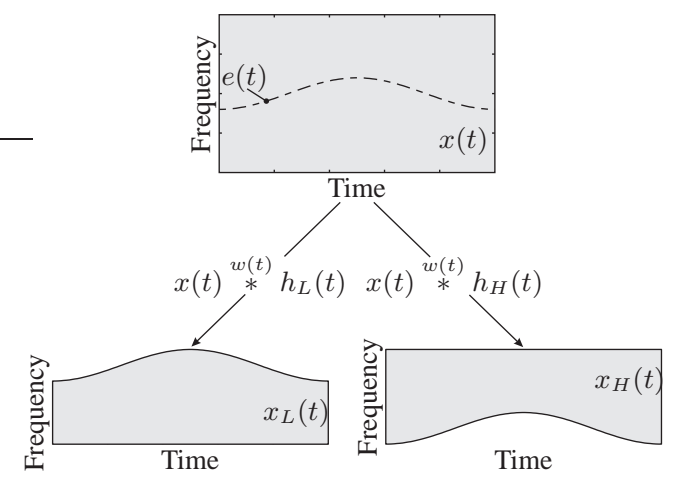

Fig. 3. Time-frequency interpretation of the class of linear timevarying filter based on non-unitary time-warping operators. Top: Received signal $x(t)$. Bottom-left: "lowpass" filtered signal. Bottom-right: "highpass" filtered signal.

This last parameter gives the dispersive behavior of the channel and is described through the modal propagation theory [9]. The impulse response $x(t)$ of dispersive channels is expressed as a sum of modes $x(t)=\sum_{n=1}^{N} h_{n}(t)$ where the mode $x_{n}(t)$ is given by :

$$
x_{m}(t)=\mathcal{F}^{-1}\left(\alpha_{m}(f) \exp \left(-j k_{r}(f, m) r\right)\right)
$$

with $\mathcal{F}^{-1}$ the inverse Fourier transform, $N$ the number of significant propagation paths, $\alpha_{m}(f)$ the attenuation of $m^{t h}$ path, $r$ the transmitter-receiver range and $k_{r}(m, f)$ a function associated to the $m^{t h}$ path. Close-form computation of (12) is a difficult task and requires the knowledge of the physical parameters of the channel. Still, a close-form expression of the group delay of $x_{m}(t)$ is available in particular cases (constant sound speed profile), leading to the following expression :

$$
\frac{1}{2 \pi} \frac{\partial k_{r}(m, f)}{\partial f}=r\left[\frac{c^{2}}{2 \pi f} \sqrt{\left(\frac{2 \pi f}{c}\right)^{2}-\left(k-\frac{1}{2}\right)^{2} \frac{\pi^{2}}{h^{2}}}\right]^{-1}
$$

where $c$ is the sound velocity, and $h$ is the transmitter depth.

To illustrate performances of the proposed characterization framework, we consider a numerical simulation of the impulse response of an underwater dispersive channel. The simulated channel is $30 \mathrm{~m}$ deep and has a rigid bottom. The distance between the source and the receiver is $1500 \mathrm{~m}$. Since the sound speed is approximatively $1500 \mathrm{~m} . \mathrm{s}^{-1}$ in water, reception instant is one second delayed from emission instant. The simulated signal is sampled at $3 \mathrm{kHZ}$, has 13000 samples, has been performed on the frequency band $[20 \mathrm{~Hz}-$ $-300 \mathrm{~Hz}$ ] and is corrupted by an additive white Gaussian noise with a $10 \mathrm{~dB}$ signal-to-noise ratio. The theoretical representation and the spectrogram of the simulated signal are displayed in the Fig. 4.

\subsection{Results}

The characterization procedure consists in the separation of each mode $x_{m}(t)$ from the signal $x(t)$ by iterating the tracking-extraction algorithm described in Sec. 2.

The first step, consists in generating a chirp sequence $\left\{\hat{x}_{m, j}(t)\right\}$ in order to estimate the instantaneous frequency of $x^{k}(t)$. The second step consists in designing a pass-band time-varying filter whose time-varying cutoff frequency matches the chirp sequence $\left\{\hat{x}_{m, j}(t)\right\}$ and extracting the mode $\hat{x}_{m}(t)$. 


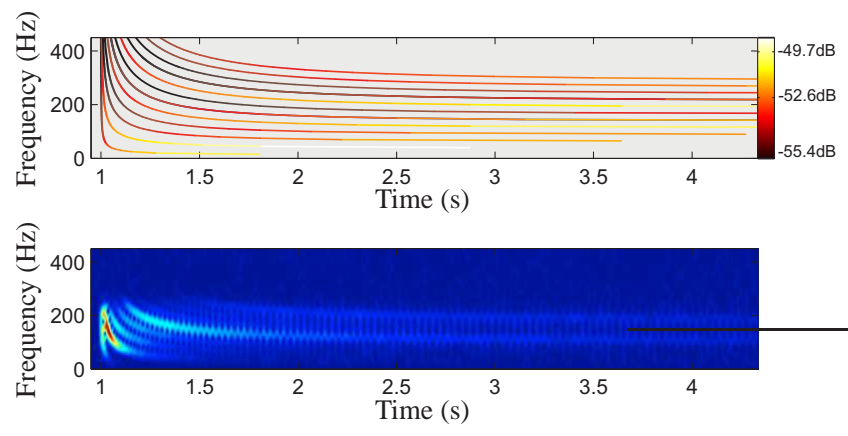

Fig. 4. Realistic numerical simulation of the impulse response of an underwater dispersive channel. Top: theoretical time-frequency representation of the channel impulse response. Each line represent a mode and the color bar represents the energy attenuation at one meter. Bottom: spectrogram of the channel impulse response.

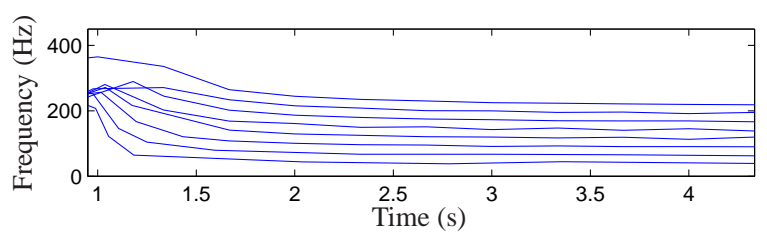

Fig. 5. Result of the time-frequency tracking step after 8 iterations. This tracks are used for the design of the non-stationary timefrequency filter.

Once the extraction of the mode $\hat{x}_{m}(t)$ has been completed, the extraction of the next mode $\hat{x}_{m+1}(t)$ is performed by the same tracking-extraction procedure on the residual signal $x(t)-\hat{x}_{m}(t)$.

The result of the tracking step after 8 iterations is depicted in Fig. 5. As can be seen, each track is clearly associated with one time-frequency component as they approximately match their instantaneous frequencies. Based on this family of tracks, it is straightforward to perform the extraction of the component by means of a passband time-varying filter.

The smoothed pseudo-Wigner-Ville distribution of the three first extracted components and the sum of the smoothed pseudo-WignerVille of the 8 extracted components are depicted in the Fig. 6. As can be seen, all highest energetic modes have been successfully extracted by the proposed tracking-extraction procedure.

However, we noticed that the proposed tracking method has difficulties to deal with quasi-vertical structures. This is explained by the fact that the discrete-time estimation of the integrals involved in the calculation of (8) is difficult due to the rapid oscillations of the cos and sin terms. This limitation can be overcome by estimating integrals in time for all $c_{i}<1$ and in the dual spectral domain for all $c_{i}>1$. This issue is left for future work.

\section{CONCLUSION}

In this paper we proposed a new time-frequency method to extract time-frequency components of the impulse response of a dispersive channel. For this purpose, we introduced a new technique based on two main features. The former is the local chirp matching using all chirp parameters. While the provided instantaneous frequency esti-

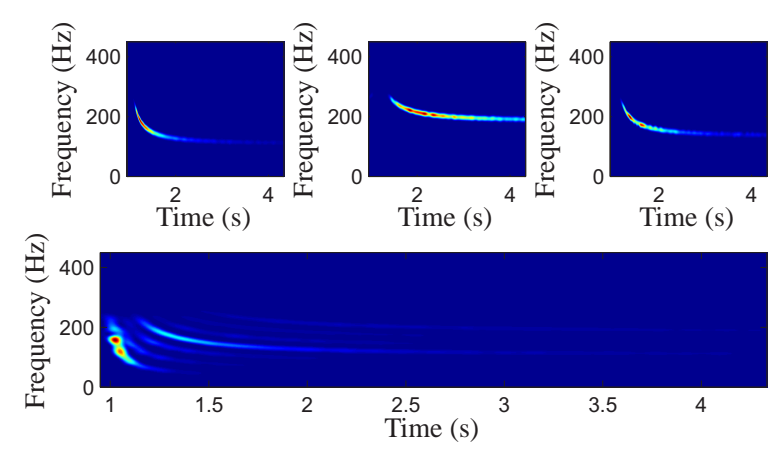

Fig. 6. Results of the tracking-extraction method.Top from left to rigth : smoothed pseudo Wigner-Ville of respectively, the first, the second and the third extracted modal arrivals. Bottom: sum of the 8 smoothed pseudo Wigner-Ville of each extracted modal arrivals.

mation is just an approximation it constitutes only an intermediary result used to design the time-frequency filter. This latter feature serves to accurately extract the corresponding signal ensuring also the conservation of both amplitude and time-frequency contents of the given structure.

Results proved the potential of the method in a realistic context from both time-frequency component proximity and amplitude. In future works we will study improvement of the proposed tracking tool by using cubic frequency modulation instead of the chirp model. This way, the tracking will achieve a better matching ratio with nonlinear time-frequency structures. In another hand, tests for real data will be conducted.

\section{REFERENCES}

[1] A. Papandreou-Suppappola, Applications in time-frequency signal processing, CRC Press, 2003.

[2] A. Papandreou-suppappola, "Dispersive channel modelling," IEEE Trans. on Signal Processing, 2006, Accepted for publication.

[3] S. Peleg and B. Friedlander, "The discrete polynomial-phase transform," IEEE Trans. on Signal Processing, vol. 43, no. 8, pp. 1901-1914, 1995.

[4] S. Mallat and Z. Zhang, "Matching pursuits with time-frequency dictionaries," IEEE Trans. on Signal Processing, vol. 41, no. 12, pp. 3397-3415, 1993.

[5] A. Jarrot, C. Ioana, and A. Quinquis, "A class of linear timevarying filters based on non-unitary time-warping operators. part i: principle and examples.," IEEE Trans. on Signal Processing, 2006, submitted for publication.

[6] I. Skolnik, Radar Handbook, 2nd edition, McGraw-Hill Professional, 1990.

[7] E. Chassande-Mottin and A. Pai, "Best chirplet chain: Nearoptimal detection of gravitational wave chirps," Physical Review, vol. 73, no. 042003, pp. 1-25, 2006.

[8] A. Jarrot, C. Ioana, and A. Quinquis, "Denoising underwater signals propagating through multi-path channels," in Oceans'05 Europe, Brest, France, June 2005.

[9] F. Jensen, W. Kuperman, M. Portor, and H. SCchmidt, Computational ocean acoustics, AIP Press, 1994, Chapter 5. 